\title{
Should asymptomatic enlarged thymus glands be resected?
}

\author{
Smit Singla, MD, MRCS (Edinburgh), MSc, ${ }^{\mathrm{a}}$ Leslie A. Litzky, MD, ${ }^{\mathrm{b}}$ Larry R. Kaiser, MD, FACS, ${ }^{\mathrm{c}}$ and \\ Joseph B. Shrager, MD $^{\mathrm{d}}$
}

Objective: Patients frequently have an "enlarged thymus" incidentally identified on imaging. We sought to determine whether thymectomy is appropriate in patients with diffusely enlarged thymus glands.

Methods: A retrospective review was conducted of patients undergoing thymectomy without myasthenia gravis at 1 institution over 15 years.

Results: Of 117 patients undergoing thymectomy, 109 patients had complete data. Thirty-six had a gland judged by the surgeon to be diffusely enlarged, and 73 had a discrete mass. Of the 36 diffusely enlarged thymus glands, $18(50 \%)$ occurred in patients with no symptoms referable to the thymus. No patient $(0 / 18 ; 0 \%)$ with an asymptomatic diffusely enlarged thymus gland had a pathologic diagnosis that would have required resection ( 8 normal; 10 "hyperplasia"). Of the 18 symptomatic patients with diffusely enlarged glands, $4(22.2 \%)$ harbored lymphoma, but none harbored thymoma or other tumor $(P<.05$; symptomatic vs asymptomatic). Of the 73 patients with discrete masses, $45(61.6 \%)$ were symptomatic, and both the symptomatic and asymptomatic patients had a high rate of pathologic diagnoses that represented an indication for resection $(53.3 \%$ and $42.8 \%$, respectively, harbored thymoma or other tumor). Of the 25 (of 109) patients initially having a diagnosis of thymic hyperplasia, only $3(12 \%)$ had true follicular hyperplasia on re-review of the pathologic condition. Interestingly, an autoimmune disorder developed in $2(67 \%)$ of these 3 patients on long-term follow-up.

Conclusions: Asymptomatic patients with diffusely enlarged thymus glands can be followed up expectantly because they have a negligible incidence of significant thymic disease; symptomatic patients with diffusely enlarged thymus glands may have lymphoma, so biopsy is appropriate. Half of patients with a discrete mass have tumors requiring resection; imaging advances would be useful to better differentiate among patients within this group. (J Thorac Cardiovasc Surg 2010;140:977-83)

Although in recent years the function of the thymus gland has come to be better defined, ${ }^{1-3}$ the association of an "abnormal" thymus with several autoimmune diseases continues to be incompletely understood. ${ }^{4}$ Among these diseases, myasthenia gravis is the most common, and substantial (though inconclusive) data have accumulated suggesting that thymectomy has a positive impact on this illness. ${ }^{5-7}$

Perhaps because of our sizeable experience with the minimally invasive procedure transcervical thymectomy in patients with nonthymomatous myasthenia gravis, ${ }^{6}$ we are often also referred a variety of patients with other suspected

\footnotetext{
From the Departments of Surgery ${ }^{\mathrm{a}}$ and Pathology and Laboratory Medicine, ${ }^{\mathrm{b}}$ University of Pennsylvania School of Medicine, Philadelphia, Pa; The University of Texas, ${ }^{\mathrm{c}}$ Houston, Tex; and the Division of Thoracic Surgery, Department of Cardiothoracic Surgery, ${ }^{\mathrm{d}}$ Stanford School of Medicine and VA Palo Alto Healthcare System, Stanford, Calif.

Read at the 36th Annual Meeting of The Western Thoracic Surgical Association, Ojai, California, June 23-26, 2010.

Disclosures: None.

Received for publication June 18, 2010; revisions received July 19, 2010; accepted for publication Aug 1, 2010.

Address for reprints: Smit Singla, MD, MRCS (Edinburgh), MSc, 233 John Morgan Bldg, 3620 Hamilton Walk, University of Pennsylvania School of Medicine, Philadelphia, PA 19104 (E-mail: smitsingla@hotmail.com).

$0022-5223 / \$ 36.00$

Copyright (c) 2010 by The American Association for Thoracic Surgery doi:10.1016/j.jtcvs.2010.08.005
}

thymic abnormalities. Among these are a substantial number of patients who do not have myasthenia gravis but are believed to have "abnormally enlarged" thymus glands without a discrete mass. These patients are often asymptomatic and the enlarged gland is often identified incidentally on a scan carried out for another reason. The finding of the suspected abnormality of diffuse thymic enlargement creates significant anxiety for both the patient and the referring physician. Although different surgeons at our institutions have taken different approaches to this problem, the overall outcome has been that a substantial number of thymus glands considered "enlarged," but without a discrete mass, have been removed.

Is this the appropriate way to manage these patients? Should they undergo thymectomy, biopsy, or simply be observed? Is there a way to decide which patients should be treated operatively and which observed expectantly?

To help resolve this set of questions, we reviewed our experience to test the following hypothesis: that a substantial portion of patients with diffusely enlarged thymus glands will harbor a pathologic process that would be an appropriate indication for surgical resection.

\section{METHODS}

We conducted a retrospective review of all patients without myasthenia gravis who underwent thymectomy at our institution between 1991 and 


\section{Abbreviation and Acronym \\ $\mathrm{CT}=$ computed tomography}

2006. The University of Pennsylvania Institutional Review Board approved the study; patients provided informed consent for telephone interview.

One hundred seventeen patients qualified for the study; of these, 109 were found to have complete records and were thus included in the analysis. Referral notes, preoperative surgeons' notes, imaging reports, operative reports, and pathologic reports were all reviewed with special attention to demographics, signs and symptoms, and the surgeon's determination of whether the thymus represented a diffusely enlarged gland versus a gland containing a discrete mass. In patients who were determined on initial pathologic diagnosis to demonstrate thymic hyperplasia, all slides were rereviewed by our most expert thymic pathologist to confirm or refute the presence of true follicular hyperplasia. Patients who had not been seen in our offices within 6 months of final data collection were contacted by telephone for up-to-date clinical follow-up.

\section{RESULTS}

Among the 109 patients, 36 (33\%) had a gland judged by the surgeon to be a diffusely enlarged thymus gland, whereas $73(67 \%)$ had a thymus containing a discrete mass. The glands were differentiated into these respective groups on the basis of computed tomographic (CT) findings. The diffusely enlarged thymus glands had a homogenous appearance, although often denser on CT scan than the fatty density of the typical involuted thymus, with preservation of normal thymic shape and contour, though judged large for age by both the radiologist and the surgeon (Figures 1 and 2). Patients with discrete thymic masses exhibited a well-defined soft tissue mass in a background of thymic tissue of different density, often altering the normal concave aspect of the junction of the gland with the mediastinal pleura (Figure 3).

There were $51(47 \%)$ male and $58(53 \%)$ female patients with a median age of 50 years (range, 12-91 years). Among the 36 patients with diffusely enlarged thymus glands, $18(50 \%)$ patients were asymptomatic at presentation and the other 18 patients had symptoms anatomically referable to the thymus, ranging from chest discomfort and pain to shortness of breath. Additionally, among patients ultimately given a diagnosis of lymphoma, 2 patients reported night sweats. In the group with discrete masses $(\mathrm{n}=73)$, only $28(38.4 \%)$ patients were asymptomatic; the remaining $45(61.6 \%)$ patients had a range of symptoms (Table 1). We subsequently analyzed the data in detail from the point of view of symptomatic versus asymptomatic patients.

For all patients, chest discomfort $(\mathrm{n}=19)$, pain $(\mathrm{n}=14)$, and shortness of breath $(\mathrm{n}=11)$ were the most common symptoms identified. Although cough and a sensation of chest fullness were found with a higher incidence, fatigue and dysphagia were seen only in patients with discrete


FIGURE 1. A, Normal thymus in a 26 -year-old patient. B, Normal thymus in a 50-year-old patient.

masses. Hoarseness was reported in only 1 patient with a diffusely enlarged thymus gland (Table 2 ).

Nearly $60 \%$ of patients $(n=65)$ were found to have abnormal histologic features on initial evaluation. Thirty patients had thymoma (diffuse enlargement $=0$, discrete mass $=30$ ), 25 patients had an initial pathologic diagnosis of hyperplasia with or without a cyst (diffuse enlargement $=17$, discrete mass $=8$ ), 5 patients had lymphoma (diffuse enlargement $=4$, discrete mass $=1$ ), and another 5 had tumors other than thymoma (diffuse enlargement $=0$, discrete mass $=5$ ) (Table 3 ). Among the other tumors, 3 were classified as germ cell tumor, metastatic renal cell carcinoma, and invasive neuroendocrine tumor, and 2 other tumors were not classified and lacked definite diagnosis.

When patients were grouped into asymptomatic and symptomatic groups, we found that none of the 18 patients with an asymptomatic diffusely enlarged thymus gland had 

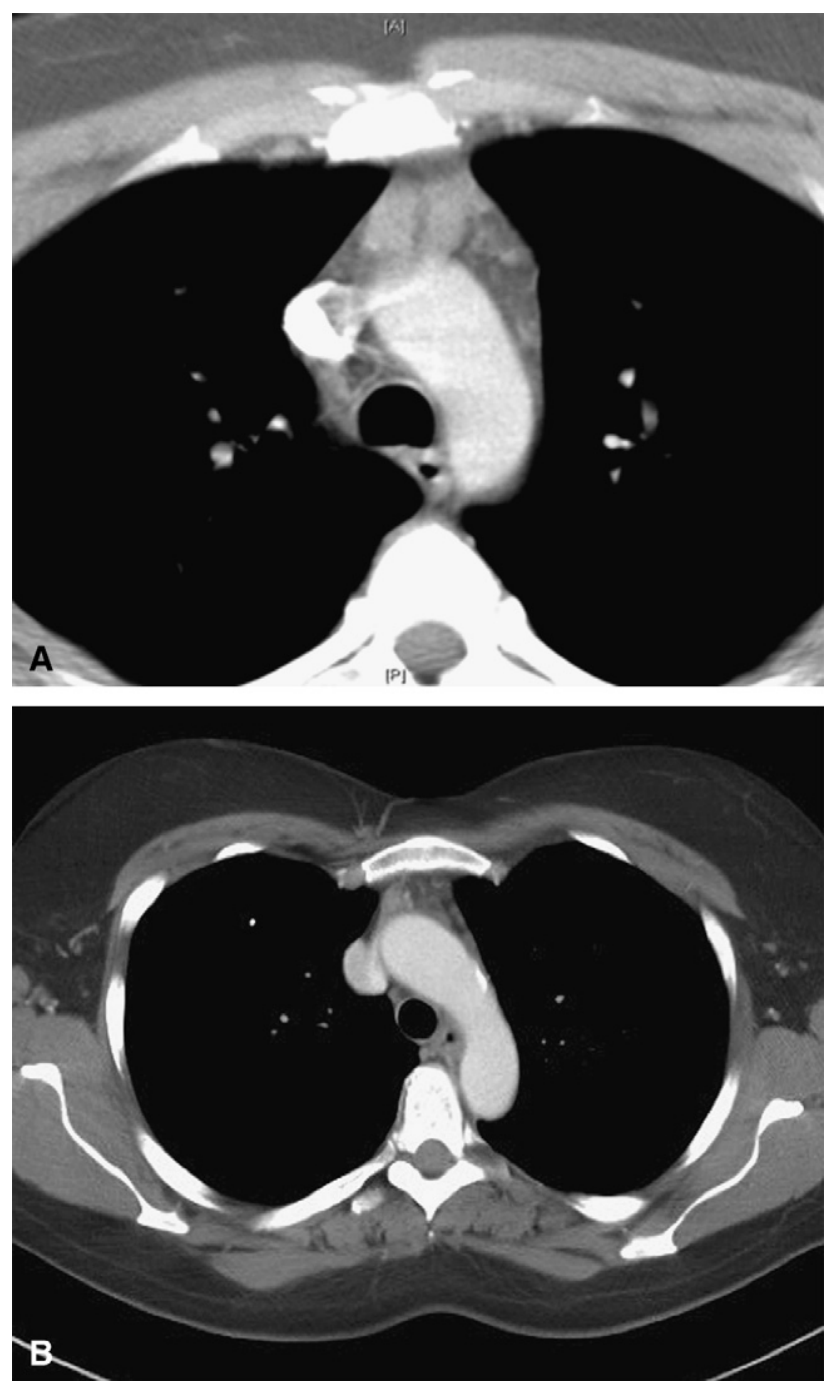

FIGURE 2. A, Diffusely enlarged thymus in a 37 -year-old patient. B, Diffusely enlarged thymus in a 50-year-old patient.

an ultimate pathologic diagnosis that would have represented an indication for resection (0/18; the maximum calculated risk of pathologic diagnosis with $95 \%$ confidence is not greater than $17 \%){ }^{8}$ Eight $(44.4 \%)$ of these patients were found to have a normal thymus with or without a small benign cyst, and the remaining $10(55.6 \%)$ patients were initially classified as having "hyperplasia" with or without a small cyst (although on re-review of the pathologic findings, only 3 patients were confirmed to have hyperplasia). Of the patients with symptomatic diffusely enlarged thymus glands, $4(22.2 \%)$ were found to harbor lymphoma in the thymus, but none harbored a thymoma or other tumor requiring resection $(P<.05$, incidence of significant diagnoses among symptomatic vs asymptomatic diffusely enlarged thymus glands) (Table 4).

As expected, in the discrete mass group, both the asymptomatic and symptomatic patients had a high rate of patho-

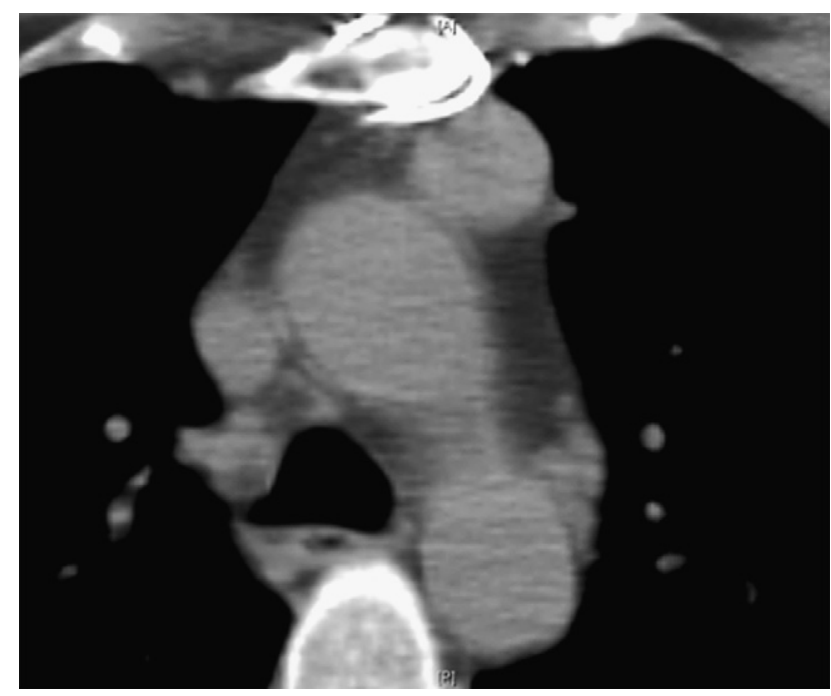

FIGURE 3. Discrete thymus mass in a 74 -year-old patient.

logic diagnoses that represented a clear indication for resection. Twelve $(42.8 \%)$ patients in the asymptomatic group and $24(53.3 \%)$ in the symptomatic group were found to have thymoma or other solid tumor, respectively (Table 4).

Of the 25 of 109 patients initially given a pathologic diagnosis of thymic hyperplasia, on re-review of the pathologic findings only $3(12 \%)$ had true follicular hyperplasia. Interestingly, on long-term follow-up, an autoimmune disorder developed in $2(67 \%)$ of these 3 patients. These included 1 case of lupus erythematosis and 1 case of ulcerative colitis. None of the other 22 patients with lesser degrees of "hyperplasia" had subsequent autoimmune disease.

\section{DISCUSSION}

Thymus glands that are, or that appear to be, abnormally enlarged clearly represent a diagnostic and therapeutic challenge for surgeons. Prior studies suggest that although preoperative imaging is reasonably successful in diagnosing discrete thymic masses, it consistently fails to differentiate between normal gland, hyperplasia, and small thymic masses. ${ }^{9-11}$ Although several studies describe the widely known relationship between age and size of the thymus gland, ${ }^{12-14}$ the algorithms that have been proposed to determine when a gland is or is not "abnormally" enlarged for a given age are sufficiently complex to be extremely difficult to apply in daily practice. ${ }^{13}$ Furthermore, there is substantial variability in the rate of thymic involution

TABLE 1. Distribution of patients based on presence or absence of symptoms

\begin{tabular}{lcc}
\hline & $\begin{array}{c}\text { Diffusely enlarged } \\
\text { thymus gland }(\mathbf{n = 3 6})\end{array}$ & $\begin{array}{c}\text { Discrete mass } \\
(\mathbf{n}=\mathbf{7 3})\end{array}$ \\
\hline Asymptomatic $(\mathrm{n}=46)$ & $18(50 \%)$ & $28(38 \%)$ \\
Symptomatic $(\mathrm{n}=63)$ & $18(50 \%)$ & $45(62 \%)$ \\
\hline
\end{tabular}


TABLE 2. Distribution of predominant symptoms among symptomatic patients

\begin{tabular}{lcc}
\hline \multicolumn{3}{c}{ Total patients with symptoms $(\mathbf{n}=\mathbf{6 3})$} \\
\hline \multicolumn{1}{c}{ Symptoms } & $\begin{array}{c}\text { Diffusely enlarged } \\
\text { thymus gland }(\mathbf{n}=\mathbf{1 8})\end{array}$ & $\begin{array}{c}\text { Discrete mass } \\
(\mathbf{n}=\mathbf{4 5})\end{array}$ \\
\hline Chest discomfort $(\mathrm{n}=19)$ & 8 & 11 \\
Pain $(\mathrm{n}=14)$ & 4 & 11 \\
Shortness of breath $(\mathrm{n}=11)$ & 2 & 9 \\
Cough $(\mathrm{n}=9)$ & 2 & 7 \\
Fullness $(\mathrm{n}=4)$ & 1 & 3 \\
Fatigue $(\mathrm{n}=3)$ & 0 & 3 \\
Dysphagia $(\mathrm{n}=1)$ & 0 & 1 \\
Hoarseness $(\mathrm{n}=1)$ & 1 & 0 \\
\hline
\end{tabular}

between individuals ${ }^{15}$ and in the size, shape, and density of thymic tissue between individuals of the same age. ${ }^{12,14}$ Inasmuch as the size of the gland can also vary with conditions like stress and inflammatory disorders, ${ }^{16}$ it remains a significant challenge to try to predict pathologic diagnoses on the basis of radiographic findings alone.

The major finding of our study is to disprove our hypothesis that a substantial portion of patients with diffusely enlarged thymus glands will harbor a pathologic process that would be an appropriate indication for surgical resection. Our finding, in fact, was nearly the opposite of this. We found that not a single patient with a diffusely enlarged thymus gland harbored a thymic tumor that required resection. The only pathologic process identified within these thymuses was lymphoma in 4 cases, which perhaps would have been more appropriately managed by biopsy than resection (though little or no harm is/was done with the resection being able to be performed by the minimally invasive procedure, transcervical thymectomy).

Additionally, all patients with diffusely enlarged thymus glands who had a diagnosis of lymphoma had symptoms referable to the thymus. Every patient who was asymptomatic and had a diffusely enlarged gland was found to have a normal gland. We believe that our findings establish, then, that the presence or absence of symptoms in patients with diffusely enlarged thymus glands is a valuable piece of information that the surgeon can use to determine whether to operate or take an expectant approach. Specifically, our findings suggest that patients with symptomatic diffusely

TABLE 3. Distribution of patients based on initial histologic characteristics

\begin{tabular}{lcc}
\hline \multicolumn{1}{c}{ Histology } & $\begin{array}{c}\text { Diffusely enlarged } \\
\text { thymus gland }(\mathbf{n}=\mathbf{3 6})\end{array}$ & $\begin{array}{c}\text { Discrete mass } \\
(\mathbf{n}=\mathbf{7 3})\end{array}$ \\
\hline Normal \pm cyst $(\mathrm{n}=44)$ & 15 & 29 \\
Hyperplasia \pm cyst $(\mathrm{n}=25)$ & 17 & 8 \\
Thymoma $(\mathrm{n}=30)$ & 0 & 30 \\
Lymphoma $(\mathrm{n}=5)$ & 4 & 1 \\
Other tumors $(\mathrm{n}=5)$ & 0 & 5 \\
\hline
\end{tabular}

TABLE 4. Pathologic diagnoses according to symptom status and indication for surgical resection

\begin{tabular}{lcc}
\hline & $\begin{array}{c}\text { Diffusely enlarged } \\
\text { thymus gland }(\mathbf{n}=\mathbf{3 6})\end{array}$ & $\begin{array}{c}\text { Discrete mass } \\
(\mathbf{n}=\mathbf{7 3})\end{array}$ \\
\hline $\begin{array}{c}\text { Asymptomatic } \\
(\mathrm{n}=46)\end{array}$ & $0 / 18^{*}$ (no significant & $12 / 28$ (thymoma/other \\
pathology seen) & solid tumor) \\
$\begin{array}{l}\text { Symptomatic } \\
(\mathrm{n}=63)\end{array}$ & $4 / 18^{*}$ (all lymphomas) & $24 / 45$ (thymoma/other \\
*Denotes $P<.05$ between asymptomatic and symptomatic diffusely enlarged thymus \\
glands.
\end{tabular}

enlarged thymus glands should undergo thymic biopsy, whereas patients without symptoms should be followed up with serial radiographic evaluation. If the thymus remains unchanged over 5 years (a rather lengthy period chosen conservatively inasmuch as thymomas can be very slowgrowing), we believe that follow-up can be terminated.

The benign thymic cysts identified within the thymic tissue in some of the patients in our study were small and subtle on preoperative imaging. Nearly all were in fact incidental findings on pathologic examination. Patients with radiographically prominent thymic cysts present a different sort of clinical challenge that has not been carefully studied. We believe that unilocular, noncalcified, thinwalled, simple fluid-density thymic cysts that are asymptomatic are highly likely to be benign and can be observed. Symptomatic cysts or cysts lacking these benign radiographic features should be resected, preferably by a transcervical or other minimally invasive approach. If any cyst grows, we believe it should be resected, and certainly any patient who chooses to have even a benign-appearing cyst observed must be counseled that there is some chance that a cystic thymoma (malignant cyst) is present.

Another interesting finding of our study is the fact that, although on re-review of pathologic characteristics true thymic follicular hyperplasia were found to be rare (only 3 cases), its presence seems in our limited sample to be highly associated with the ultimate development of some type of autoimmune disease. Such a process ultimately developed in 2 of the 3 patients with true follicular hyperplasia. This suggests a pathophysiologic relationship between thymic follicular hyperplasia and autoimmune disorders. This is obviously consistent with the widely known association of thymic abnormalities with myasthenia gravis, but also with the less widely known association of thymic abnormalities with other autoimmune disorders ${ }^{17-19}$ and with the association of thymectomy with resolution in some of these disorders. ${ }^{19,20}$ We believe that additional prospective study of thymectomy as a therapy for the more common of these autoimmune disorders would be reasonable.

In distinct contrast to patients with diffusely enlarged thymus glands, nearly half of all patients with discrete masses proved to have tumors that should be resected. An alternative way of looking at this, however, is that 
approximately $50 \%$ of patients with discrete masses will be found to have normal thymic pathologic conditions and so will have undergone what might be termed an unnecessary procedure. The presence or absence of symptoms was not found to be useful in selecting patients with discrete thymic masses requiring an intervention as it appears to be in patients with diffusely enlarged thymus glands.

With regard to a role for functional imaging, the available evidence suggests that positron emission tomographic scanning has only marginal utility in differentiating benign from malignant processes within the thymus. ${ }^{21,22}$ At our institutions, we do not employ positron emission tomographic scans to aid in the differential diagnosis of enlarged thymus glands unless there is a history of or clinical suspicion of lymphoma, which is generally markedly fluorodeoxyglucose-avid. Future refinements in nuclear tumor imaging or other radiologic techniques that may help to delineate processes that should/should not be resected would represent major advances in this field.

Although this study is obviously limited by its retrospective nature and its modest sample size, we believe that the perfect correlation that we have found between an asymptomatic presentation and the discovery of benign pathologic conditions in diffusely enlarged thymus glands is sufficiently impressive to guide management decisions. We recommend that patients with diffusely enlarged thymus glands and no symptoms referable to the thymus undergo observation with serial CT scanning while those with symptoms undergo thymic biopsy.

We thank Makoto Katsumata, $\mathrm{PhD}$, for his contribution to statistical analyses.

\section{References}

1. Mori T, Nomori H, Ikeda K, Kobayashi H, Iwatani K, Kobayashi T. The distribution of parenchyma, follicles, and lymphocyte subsets in thymus of patients with myasthenia gravis, with special reference to remission after thymectomy. J Thorac Cardiovasc Surg. 2007;133:364-8.

2. Yarilin AA, Belyakov IM. Cytokines in the thymus: production and biological effects. Curr Med Chem. 2004;11:447-64.

3. Hadden JW. Thymic endocrinology. Ann N Y Acad Sci. 1998;840:352-8.

4. Irvine WJ. The thymus in autoimmune disease. Proc $R$ Soc Med. 1970;63: 718-22.

5. Jaretzki A 3rd, Penn AS, Younger DS, Wolff M, Olarte MR, Lovelace RE, et al. "Maximal" thymectomy for myasthenia gravis. Results. J Thorac Cardiovasc Surg. 1988;95:747-57.

6. Shrager JB, Nathan D, Brinster CJ, Yousuf O, Spence A, Chen Z, et al. Outcomes after 151 extended transcervical thymectomies for myasthenia gravis. Ann Thorac Surg. 2006;82:1863-9.

7. Mack MJ, Landreneau RJ, Yim AP, Hazelrigg SR, Scruggs GR. Results of videoassisted thymectomy in patients with myasthenia gravis. $J$ Thorac Cardiovasc Surg. 1996;112:1352-9.

8. Hanley JA, Lippman-Hand A. If nothing goes wrong, is everything all right? Interpreting zero numerators. JAMA. 1983;249:1743-5.

9. Nicolaou S, Müller NL, Li DK, Oger JJ. Thymus in myasthenia gravis: comparison of $\mathrm{CT}$ and pathologic findings and clinical outcome after thymectomy. Radiology. 1996;201:471-4.

10. Pirronti T, Rinaldi P, Batocchi AP, Evoli A, Di Schino C, Marano P. Thymic lesions and myasthenia gravis. Diagnosis based on mediastinal imaging and pathological findings. Acta Radiol. 2002;43:380-4.
11. Yasukawa Y, Yoshikawa H, Iwasa K, Yamada M, Takamori M. Comparative study of preoperative thymic imaging and pathology in patients with myasthenia gravis. J Clin Neurosci. 2004;11:610-3.

12. Baron RL, Lee JK, Sagel SS, Peterson RR. Computed tomography of the normal thymus. Radiology. 1982;142:121-5.

13. Francis IR, Glazer GM, Bookstein FL, Gross BH. The thymus: reexamination of age-related changes in size and shape. AJR Am J Roentgenol. 1985;145: 249-54.

14. Moore AV, Korobkin M, Olanow W, Heaston DK, Ram PC, Dunnick NR, et al Age-related changes in the thymus gland: CT-pathologic correlation. AJR Am J Roentgenol. 1983;141:241-6.

15. Bogot NR, Quint LE. Imaging of thymic disorders. Cancer Imaging. 2005;5: 139-49.

16. Nishino M, Ashiku SK, Kocher ON, Thurer RL, Boiselle PM, Hatabu H. The thymus: a comprehensive review. Radiographics. 2006;26:335-48.

17. Tsuchiya M, Asakura H, Yoshimatsu H. Thymic abnormalities and autoimmune diseases. Keio J Med. 1989;38:383-402.

18. Shimabukuro K, Mizuno Y, Kurita K, Tsuchiya M, Yoshimatsu H. Thymus in Hashimoto's disease and focal chronic lymphoid thyroiditis. Keio J Med. 1975;24: 275-87.

19. Tsuchiya M, Hibi T, Watanabe M, Ohara M, Ogata H, Iwao Y, et al. Thymectomy in ulcerative colitis: a report of cases over a 13 year period. Thymus. 1991;17: 67-73.

20. Yoshimatsu H, Ishikura Y, Odagiri S, Mizoguchi Y. Clinical examinations of thymic abnormalities and significance of thymectomy in the patients with autoimmune disease. Rinsho Kyobu Geka. 1989;9:267-72.

21. Luzzi L, Campione A, Gorla A, Vassallo G, Bianchi A, Biggi A, et al. Role of fluorine-flurodeoxyglucose positron emission tomography/computed tomography in preoperative assessment of anterior mediastinal masses. Eur J Cardio thorac Surg. 2009;36:475-9.

22. Nakahara T, Fujii H, Ide M, Nishiumi N, Takahashi W, Yasuda S, et al. FDG uptake in the morphologically normal thymus: comparison of FDG positron emission tomography and CT. Br J Radiol. 2001;74:821-4.

\section{Discussion}

Dr Michael Weyant (Aurora, Colo). I thank the authors for providing me with the manuscript and slides well ahead of the deadline. I enjoyed the paper very much.

Dr Singla, you and your colleagues have been able to address a clinical scenario that is frequently encountered by thoracic surgeons. Until now, we have had no concrete recommendations about how to deal with the problem. I think your series represents the largest published to date regarding this phenomenon. The most interesting aspect of your presentation deals with the diffusely enlarged thymus, and I have a few questions.

Your data state that 4 of the 36 patients who had resection of a diffusely enlarged thymus had abnormal pathologic features, and they were lymphomas in all 4 of the cases. Inasmuch as 4 of the patients were symptomatic and in half the patients who had no symptoms no abnormal pathologic condition was demonstrated, you recommend that if a patient has no symptoms and a diffusely enlarged thymus, he or she can be monitored without resection. I would like to play the devil's advocate: Most of us find it very difficult to attribute the true symptoms of the patient to the thymus; the symptoms are very nonspecific and unreliable. Therefore, you could use that as one of the limitations of your study in gauging whether the patient's symptoms are truly attributable to the thymus. In that setting, if you look at the other side of the argument, you would say that in 4 of these 36 patients who have a diffusely enlarged thymus, greater than $10 \%$ of the time you find abnormal pathologic features. You actually may be able to submit an opposite conclusion-maybe resection should be 
done in all those patients because the symptoms of these patients are so unreliable. May I have your comments on that?

Dr Singla. You are correct that 4 of the patients with diffusely enlarged thymus glands and symptoms actually had lymphoma, and our recommendation is that for patients with lymphoma biopsy is probably preferable (since it is less morbid) to resection. In most instances, a CT-guided core needle biopsy will be sufficient, but if more tissue is needed a Chamberlain procedure can be done. Having said this, for the rest of the patients-those who clearly have no symptoms-our recommendation is to wait and watch. One could wait for 3 to 6 months and then scan them again with little risk of missing anything. It is unlikely that even thymoma will become metastatic in 3 to 6 months. If the first scan is unchanged, one could then do a scan yearly after that for about 5 years. Our data support the theory that nearly all of those patients will never need surgery.

I think that one should have a low threshold to biopsy if any symptoms are present that can be related in any way to the thymus. This would include any lymphoma-type symptoms (for example, "B" symptoms) or symptoms that can result from compression of structures around the thymus (such as cough or chest pain).

Dr Weyant. My second comment relating to your biopsy point is that it might be wiser to resect the thymus rather that doing a biopsy in these patients because of the amount of tissue required and the fact that it is quite difficult to get adequate biopsy tissue. All of the abnormal pathologic features you have demonstrated were lymphoma.

Dr Singla. I shall ask Dr Shrager, my senior surgeon, to respond because he has more experience doing these operations. Let me ask him for some comments.

Dr Shrager. If you can do a transcervical thymectomy-if you know how to do that - then maybe it is not unreasonable to do a thymectomy in the case of a symptomatic gland, because it probably is just as benign a procedure as doing a Chamberlain procedure. If you have pathologists who are comfortable, with good flow cytometry, in making a diagnosis of lymphoma on the basis of a needle biopsy, then a core needle would probably be the way to go in one of these symptomatic glands.

Dr Weyant. In looking at the patients in whom you re-reviewed the pathologic features for thymic hyperplasia, you found that in all except 3 the diagnosis was refuted and changed after re-review. What is the reasoning behind that? Why do you think after the rereview the diagnosis was actually changed?

Dr Singla. That is a very good question. In discussing thymic hyperplasia, a lot of pathologists who do not have in-depth experience with thymic diseases will call any gland with increase in the weight or the size of the gland "hyperplastic." On the first time around in pathology, when a pathologist other than our key thymic pathologist often reviewed the cases, the thymuses that were enlarged but did not actually demonstrate histologic follicular hyperplasia were often classified as "thymic hyperplasia." When we went back, we actually went more in depth into the histologic characteristics and found that most of those patients did not demonstrate true histologic thymic follicular hyperplasia. There is a difference between follicular hyperplasia (which is a histologic finding) and simple enlargement of the gland. It is actually the follicular hyperplasia that has been closely associated with autoimmune diseases, and that was one of the reasons that we went back to see whether these thymic hyperplasias were actual examples of follicular hyperplasia or not.

Dr Robert Cerfolio (Birmingham, Ala). Are you sure you studied the right patients, or should you have studied patients like the 45 or 50 I have in my practice that I have not operated on and follow up every year with a CT scan?

Dr Singla. I think we did our best to study these patients in detail. We did go into depth in reading all the notes and talking with these patients.

Dr Cerfolio. Did you study any of the patients who did not get operated on?

Dr Singla. No, sir. These patients were all patients who had been operated on.

Dr Cerfolio. Are you sure you studied the right patients or should you go back and look at patients with enlarged glands who have been followed up and have never undergone surgical resection and see how they did?

Dr Singla. That is a very good suggestion. We can do that, but at present in the current data I only have patients that were operated on.

Dr Cerfolio. I do not think that any new technology that makes an operation less minimally invasive or easier to do or to recover from should change the indication for surgery. For example, I am doing lots of robotic thymectomies now. They are fun and the patients go home the next day. You had mentioned that the fact that you can do these from the neck might influence your indication. Do you think that is true or should it be the patient's anxiety that influences your decision, or the anatomic features of the gland itself, and not the surgical approach?

Dr Singla. Dr Cerfolio, I did not say that doing transcervical thymectomy should influence our indications. I said that in the past it may have influenced our decision before we knew that it was safe to leave many of these untouched. With the results of this study, I think we can now see that many of the patients in whom we performed thymectomy, partly because of the availability of this simple approach, could safely have been left without surgical intervention.

However, patients do have anxiety, especially when they have been referred and told that they have a mass. Anybody in this audience who was told that he or she had a mass would want answers, so a lot does have to do with patient anxiety. In the scenario that you have stated, that you have more advanced technology, yes, we can definitely go in and operate on these patients safely. At the same time, if we do not need to operate on these patients, then we should not.

Dr Cerfolio. You sound like a great politician, so let me try to pin down your position. Does the fact that you can do an operation minimally invasively change your indication for surgery: yes or no?

Dr Singla. I would prefer to ask Dr Shrager to answer that question, but I would say no.

Dr Frederic Grannis (Duarte, Calif). Thank you for doing this study. It gives very good information on a common problem. We know from the data from the leukocytapheresis (LCAP) study that if you use a CT scan to screen people for lung cancer, you find at least 3 thymic enlargements in 1000 patients, maybe more. Thus, this is a common problem that we need to learn how to manage.

I want to ask you 2 specific questions. First, did you do positron emission tomographic scans on any of these patients? Does 
positron emission tomography help to distinguish a thymoma from a nonthymoma?

Second, recently we avoided surgery in a patient with diffuse mass in the thymus because of hyperthyroidism. After that patient was treated for hyperthyroidism, the thymic hyperplasia resolved.

Dr Singla. Thank you, sir. With regard to your first question, there have been some small studies using positron emission tomographic scans in trying to read thymomas and other thymic disorders. Some of these studies have suggested that such scans can be useful. After discussing this with my colleagues and senior colleagues, we believe that the data are not robust enough to say that the positron emission tomographic scan alone would be sufficient to give us the correct indication or correct information. I think it is a combination of what we are suggesting is a combina- tion; a combination of signs and symptoms along with the CT scan would be more appropriate at this stage in helping make the decision on how to proceed. In the coming years, with more information coming, I think the positron emission tomographic scan may add a lot of information. A negative positron emission tomographic scan would certainly be reassuring in patients on whom you decide to follow up rather than operate.

Second, as you mentioned with the thyroid disorders, there are clearly several diseases that are widely known to cause thymic enlargement, and being aware of these is an important way to avoid unnecessary thymectomy. Besides Graves disease, another common one is postchemotherapy rebound enlargement of the thymus. I am not sure whether these represent actual follicular hyperplasia or just enlargement, but they generally resolve with time or with resolution of the underlying problem (eg, hyperthyroidism). 